\title{
Saliva as a Diagnostic Fluid for Neurological Disorder
}

\author{
Kumari $S^{1}$, Kumaran $S^{1}$ and Goyal $\mathrm{V}^{2^{*}}$ \\ ${ }^{1}$ Departments of NMR and MRI facility, All India Institute of Medical Sciences, New Delhi, India \\ ${ }^{2}$ Departments of Neurology, All India Institute of Medical Sciences, New Delhi, India
}

*Corresponding author: Goyal V, Departments of Neurology, All India Institute of Medical Sciences, New Delhi, India, Tel: + 9810470250, E-mail: drvinaygoyal@gmail.com

Citation: Kumari S, Kumaran S, Goyal V (2019) Saliva as a Diagnostic Fluid for Neurological Disorder. J Neurosci Neuropsyc 3: 103

Article history: Received: 27 May 2019, Accepted: 27 June 2019, Published: 28 June 2019

\begin{abstract}
Existing research on neurological disorders is emphasized on finding novel biomarkers to help in diagnosis, provide biochemical information and in monitoring the disease progression. Saliva has appeared as a diagnostic fluid for the detection of disease biomarkers in genomics, proteomics and metabolomics approach. Saliva offers several advantages over other biological fluids as it is a simple way of the collection that does not require expert training, painless to the patient and non-invasive. Here, study reviews the existing literature on saliva based on metabolomics study and examine their potential in diagnosing and monitoring neurodegenerative disorder such as Parkinson's, Alzheimer's, Dementia and Multiple sclerosis. Based on the available research several metabolites such as tau protein, lactoferrin, DJ1protein, histamine, propionate, acetic acid, glycerol, succinate, taurine, dimethyl sulfone, oligomeric a-synuclein, alfa-amyloid, sphinganine-1-phosphate, ornithine, phenyllactic acid, thiobarbituric acid, arginine, tyrosine and taurine that demonstrate as reliable disease biomarkers.
\end{abstract}

Keywords: Saliva; Body Fluids; Metabolomics; Parkinson's Disease; Alzheimers's Disease; Dementia

List of abbreviations: AD: Alzheimer disease; A 342 : Amyloid beta 42; MALDI/TOF-MS: Matrix Assisted Laser Desorption/Ionization/ Time-of-Flight-Mass Spectrometry; CE-TOF-MS: Capillary Electrophoresis Time-of-Flight Mass Spectrometry; CSF: Cerebrospinal fluid; ELISA: Enzyme-linked immunosorbent assay; GABA: Gamma-aminobutyric acid; GFC: Gel filtration chromatography; NDD: Neurodegenerative disease; MS: Multiple sclerosis; MCI: Mild Cognitive Impairment; LC-MS: Liquid chromatography-mass spectrometry, I3DCH: Inosine 3-Dehydrocarnitine Hypoxanthine; NMR: Nuclear magnetic resonance spectroscopy; PD: Parkinson disease; TCA: Tri-carboxylic cycle; FUPLC-MS: Fast ultra-high performance liquid chromatography coupled with time-of-flight mass spectrometry

\section{Introduction}

Early diagnosis of neurological disorders is critical to prevent complications and negative impact on the quality of life of patients. The use of potential biomarkers has great importance for the prediction, diagnosis, and monitoring the treatment effects of many diseases; hence there is an urgent need for sensitive and specific biomarker(s). Due to various factors including lack of awareness, resource deficit, and social circumstances, several diseases are misdiagnosed until a late phase. To deal with these problems, researchers are investigating biomarkers that reflect the pathological state of an individual that helps in understanding the underlying cause of the disease [1].

To understand the pathogenesis and biochemistry of neurodegenerative disease and its complexities, new ideas and new technologies are much needed. Over the last decades, studies have revealed that perturbations in human genetics, alterations in nucleic acids and proteins of the patient's bodily fluid such as blood, urine and cerebrospinal fluid (CSF), which can be used as potential biomarkers for disease diagnosis [2-4]. Compared with blood or CSF, saliva offers distinct advantages for diagnostic or research purposes; it is also possible to monitor the general population with disease. Currently, attention has been focused on the potential of saliva as a diagnostic fluid in terms of and disease diagnosis and monitoring the disease severity [5-8]. Saliva comprises biomarkers which can be used in the clinical diagnosis of disease and in pre-clinical research [9]. A biomarker is an indicator of biological process and is a characteristic that is accurately measured and assessed the pathogenic process, treatment response as per the National Institutes of Health (NIH). According to NIH, a biomarker needs to be verified and validated before its application in clinical practice and must any impact or application in health risk assessment [10,11]. Identification and utility of saliva as bio-fluid and its markers are still in its development phase, especially in conditions like neurological disorders, because, the brain and saliva are not closely related anatomically [12].The main purpose of the salivary biomarker studies is to identify 
specific biomarkers for the diagnosis of neurodegenerative diseases (NDD). It is the heterogeneous group of conditions in which the central nervous system is disrupted and affects various aspects of daily functioning such as problems in motor function, neuropsychiatric problems, learning, or communication [13]. Because NDD is heterogeneous in nature, finding a single biomarker relative to one type of disease is difficult. Thus, metabolite identified by metabolomics study is considered feasible and would be reliable biomarkers for the disease. This could also be useful in understanding the biochemistry of the disease by knowing the biological process of individual metabolites and their correlation with the metabolic pathways involved in the disease. It may help the neurologists and pharmacologist in better clinical management. The present review focused on physiology and applications of saliva as a diagnostic fluid for NDD. The related study in saliva using metabolomics techniques for NDD are summarized (Table 1).

\begin{tabular}{|c|c|c|c|c|c|c|c|}
\hline \multirow[b]{2}{*}{ References } & \multirow[b]{2}{*}{ Disease } & \multirow[b]{2}{*}{ Patient } & \multirow[b]{2}{*}{ Control } & \multirow[b]{2}{*}{ Method } & \multicolumn{2}{|c|}{ Potential biomarkers } & \multirow[b]{2}{*}{ Synopsis of result } \\
\hline & & & & & Up-regulated & Down-regulated & \\
\hline Huan, et al. 2018 & $\begin{array}{l}\mathrm{MCI}, \\
\mathrm{AD}\end{array}$ & 35 & 42 & LC-MS & $\begin{array}{l}\text { Methylguanosine, } \\
\text { Histidinyl- } \\
\text { Phenylalanine, } \\
\text { Choline-cytidine, } \\
\text { Glucosylgalactosyl } \\
\text { Hydroxylysine, } \\
\text { Glutamine-carnitines }\end{array}$ & & $\begin{array}{l}\text { Higher metabolites } \\
\text { level may distinguish } \\
\text { AD from CN and } \\
\text { MCI with good } \\
\text { diagnostic ability }\end{array}$ \\
\hline Carro et al, 2017 & $\begin{array}{l}\mathrm{AD} \\
\mathrm{MCI}\end{array}$ & $\begin{array}{l}80 \\
59\end{array}$ & 91 & MALDI-TOF/TOF-MS & & Lactoferrin & $\begin{array}{l}\text { Lower lactoferrin may } \\
\text { detect patients with } \\
\text { very early stages of } \\
\text { MCI and AD }\end{array}$ \\
\hline Yilmaz, et al. 2017 & $\begin{array}{l}\mathrm{MCI} \\
\mathrm{AD} \\
\mathrm{HC}\end{array}$ & $\begin{array}{l}8 \\
9\end{array}$ & 12 & NMR & $\begin{array}{c}\text { Imidazole, } \\
\text { Acetone, Creatine, } \\
\text { 5-Aminopentanoate, } \\
\text { Propionate, and } \\
\text { Acetone }\end{array}$ & Galactose, & $\begin{array}{c}\text { Altered metabolites } \\
\text { may predict early AD } \\
\text { and MCI }\end{array}$ \\
\hline $\begin{array}{c}\text { Figueira, et al. } \\
2016\end{array}$ & Dementia & 49 & 94 & NMR & $\begin{array}{l}\text { Histamine, Propionate, } \\
\text { Acetic Acid }\end{array}$ & $\begin{array}{c}\text { Glycerol, } \\
\text { Succinate, } \\
\text { Taurine, } \\
\text { Dimethyl sulfone }\end{array}$ & $\begin{array}{c}\text { Impairment in } \\
\text { taurine/ hypotaurine } \\
\text { pathway, pyruvate } \\
\text { metabolism, } \\
\text { glycolysis, and TCA } \\
\text { cycle and suggested } \\
\text { saliva metabolome } \\
\text { may predict dementia } \\
\text { in early stages }\end{array}$ \\
\hline Kang, et al. 2016 & $\mathrm{PD}$ & 201 & 67 & GFC and Western blot & Oligomeric $\alpha$-Synuclein & & $\begin{array}{l}\text { A higher level of } \\
\text { a-Synuclein may } \\
\text { serve as a potential } \\
\text { biomarker for PD }\end{array}$ \\
\hline Liang, et al. 2015 & $\mathrm{AD}$ & 256 & 218 & FUPLC and MS & $\begin{array}{c}\text { Alfa-Amyloid } \\
\text { Protein, Sphinganine- } \\
\text { 1-phosphate, } \\
\text { Ornithine, Phenyllactic } \\
\text { acid }\end{array}$ & $\mathrm{I} 3 \mathrm{DCH}$ & $\begin{array}{c}\text { Alterations in these } \\
\text { metabolites may } \\
\text { detect early stages } \\
\text { of } \mathrm{AD}\end{array}$ \\
\hline Martin, et al. 2015 & $\begin{array}{l}\text { Multiple } \\
\text { sclerosis }\end{array}$ & 29 & 29 & spectrophotometry & Thiobarbituric acid & & $\begin{array}{l}\text { A higher level of } \\
\text { thiobarbituric acid } \\
\text { which is the oxidative } \\
\text { stress marker may } \\
\text { predict the disease } \\
\text { severity of MS } \\
\text { patients }\end{array}$ \\
\hline Kang, et al. 2014 & $\mathrm{PD}$ & 285 & 91 & $\begin{array}{c}\text { quantitative and } \\
\text { sensitive Luminex assay }\end{array}$ & Dj1 & & $\begin{array}{l}\text { Higher DJ-1 protein } \\
\text { may serve as a } \\
\text { potential biomarker } \\
\text { for PD }\end{array}$ \\
\hline $\begin{array}{c}\text { Tsuruoka, et al. } \\
2013\end{array}$ & $\overline{\text { Dementia }}$ & 10 & 9 & CE-TOF-MS & & $\begin{array}{l}\text { Arginine and } \\
\text { Tyrosine }\end{array}$ & $\begin{array}{l}\text { Impairment in redox } \\
\text { stress, inflammatory } \\
\text { process, regulation } \\
\text { of synaptic plasticity, } \\
\text { neurogenesis, and } \\
\text { modulation of } \\
\text { glucose metabolism } \\
\text { may be helpful in } \\
\text { understanding the } \\
\text { pathology of dementia }\end{array}$ \\
\hline
\end{tabular}




\begin{tabular}{|c|c|c|c|c|c|c|c|}
\hline \multirow{2}{*}{ References } & \multirow{2}{*}{ Disease } & \multirow{2}{*}{ Patient } & \multirow{2}{*}{ Control } & \multirow{2}{*}{ Method } & \multicolumn{2}{|c|}{ Potential biomarkers } & \multirow{2}{*}{ Synopsis of result } \\
\hline & & & & & Up-regulated & Down-regulated & \\
\hline Zheng, et al. 2012 & $\mathrm{MCI}$ & 20 & 20 & LC-MS & & Taurine & $\begin{array}{l}\text { Perturbation in } \\
\text { taurine and hypo } \\
\text { taurine pathways }\end{array}$ \\
\hline Devic, et al. 2011 & $\mathrm{PD}$ & 24 & 25 & Immunoblotting & $\mathrm{Dj} 1$ & a-Synuclein & $\begin{array}{l}\text { Identified } \alpha \text {-Syn and } \\
\text { DJ1 may predict PD. } \\
\text { Association of } \alpha \text {-Syn } \\
\text { pathology with the } \\
\text { dry mouth of PD } \\
\text { patients }\end{array}$ \\
\hline Pareja, et al. 2010 & $\mathrm{AD}$ & $\begin{array}{l}70 \mathrm{AD} \\
51 \mathrm{PD}\end{array}$ & 56 & ELISA & $\mathrm{A} \beta 42$ & & $\begin{array}{c}\text { A higher level of } \mathrm{A} \beta \\
42 \text { may detect } \mathrm{AD} \text { at } \\
\text { an early stage }\end{array}$ \\
\hline
\end{tabular}

Table 1: Salivary biomarkers identified for neurodegenerative diseases

\section{Physiology of saliva}

Saliva is an aqueous fluid containing organic and inorganic molecules secreted by the salivary glands and other substances which are coming from the oropharynx, upper airway, gingival sulcus fluid, gastrointestinal reflux, food deposits [14]. The inorganic part of saliva composed of weak and strong ions, with the most important being $\mathrm{Na}^{+}, \mathrm{K}^{+}, \mathrm{Cl}, \mathrm{Ca}_{2}^{+}, \mathrm{HCO}_{3}, \mathrm{Mg}^{2+}$, and $\mathrm{NH}_{3}$ and organic part contains urea, uric acid and creatinine, cholesterol and fatty acids, and more than 400 types of protein [15]. Excretion of salivary fluid and protein molecules is controlled by autonomic nerves supplied by cholinergic parasympathetic nerves which release acetylcholine evoking the secretion of saliva by acinar cells in the end pieces of the salivary gland ductal tree [16]. Most of the salivary glands obtain a variable innervation from sympathetic nerves which releases noradrenaline and stored proteins from acinar and ductal cells [17]. In addition, saliva plays a vital role in esophageal physiology, the digestive system and gastrointestinal [15]. Evaluation of salivary metabolic profile has become an important source for the assessment of the physiological and pathological state of the individual and is also a useful tool for disease diagnosis and prognosis mainly due to its connections with other organ systems. Moreover, saliva is used as a diagnostic fluid due to its exchange with affluence that contains the plasma and this occurs through the thin layer of epithelial cells by active carriage, through diffusion, or through passive diffusion [18].

\section{Advantages of saliva as a diagnostic fluid}

Saliva as a diagnostic fluid offers characteristic advantages over blood or CSF because 1) Non-invasive collection, 2) Easy to collect by modestly trained assistant and applicable in remote areas, 3) Samples can be obtained at several times, 4) Self-collection by subject, at home, 5) Relatively cheap technique in comparison with other tests, 6) Needs less manipulation during diagnostic processes compared to serum, 7) Painless to the patient. All these features make saliva as an interesting diagnostic fluid for the detection and monitoring the disease with infants, children, adults and uncooperative patients [19].

\section{Metabolomics study of saliva in NDD}

\section{Parkinson's Disease}

In Parkinson's disease (PD) there is a selective loss of dopaminergic cells in the substantia nigra and other related neuronal systems [20]. PD is triggered by several causative monogenetic mutations which only explain about 15\% of all PD [21]. Biomarker discovery in PD has conventionally been focused on neuroimaging techniques; therefore it holds a substantial time as well as too expensive for the patient. Currently, the pathological mechanism of PD remains unclear and there are no effective biomarkers for PD diagnosis. Therefore, biomarkers are greatly needed in clinical practice that can improve traditional symptom-based evaluation and prediction of treatment response.

In order to search biomarkers of PD, Kang, et al. compared salivary metabolome of $201 \mathrm{PD}$ and 67 healthy controls using Gel filtration chromatography (GFC) and Western blot, they found the significantly increased levels of oligomeric $\alpha$-synuclein in PD as compared to controls. Analysis indicated that oligomeric a-synuclein could be used as a potential diagnostic indicator of PD [22]. Another study by Devic, et al. evaluated salivary metabolites of 24 patients with PD and control subjects using the immunoblotting technique. The analysis identified $\alpha$-Syn and DJ1 as potential biomarkers for the diagnosis of PD and providing the relevance of using saliva as a diagnostic fluid. The study suggested that $\alpha$-Syn pathology within the parasympathetic/ sympathetic innervations of salivary glands might contribute to the occurrence of dry mouth in PD [23]. In addition, salivary metabolomics was also used for PD biomarker identification. Salivary metabolomics of 285 patients with PD and 91 healthy controls were analyzed using quantitative and sensitive Luminex assay method. The DJ-1 protein was selected as a potential biomarker and their correlation with striatal dopaminergic function for PD, suggesting salivary DJ-1, indicating a potential value in the diagnosis of PD [7]. 


\section{Dementia}

Dementia is becoming a major public health challenge with a heavy economic burden, approximately 46 million people worldwide affected with dementia in 2015. Thus, it urgently needs to understand dementia pathology and identification of biomarkers for predicting the risk of dementia in the preclinical phase for preventing, monitoring, and treatment. Metabolomics technology (OMICS) provides a novel methodology for the identification of biomarkers for patients with dementia. Tsuruoka, et al. analyzed serum and saliva metabolome in 10 dementia patients and 9 age-matched healthy controls using capillary electrophoresis time-of-flight mass spectrometry (CE-TOF-MS). The analysis revealed significantly different metabolites: six in serum ( $\beta$-alanine, creatinine, hydroxyproline, glutamine, iso-citrate, and cytidine) and two in saliva (arginine and tyrosine) suggesting the impairment in redox stress, inflammatory process, regulation of synaptic plasticity, neurogenesis, and modulation of glucose metabolism. The study concluded that salivary metabolic profiles are associated with many physiological and environmental factors [24]. Zheng, et al. analyzed the saliva samples in patients with mild cognitive impairment (MCI) and healthy controls using liquid chromatography-mass spectrometry (LC-MS) technique. The study found reduced levels of taurine in MCI patients as compared to controls suggesting taurine and hypo taurine pathway may play an important role in the pathophysiology of MCI [8]. The correlation between these potential metabolites and dementia must also be further explored to understand the pathogenesis and therapeutic targets.

\section{Alzheimer disease}

$\mathrm{AD}$ is a chronic NDD, results from aggregation and accumulation of amyloid-beta in the brain becoming more prevalent in which aging is a major risk factor affecting about 26 million to more than 100 million worldwide [25]. The early diagnosis requires a better understanding of the physiological mechanisms involved before the mental decline has occurred. Therefore there is a serious need for convenient and innovative approaches to drug development and evaluation. Despite the increasing global prevalence, the precise pathogenesis and terms for objective diagnosis of neurodegenerative dementias remain controversial and comprehensive understanding of the disease remains lacking. The previous study by Carro et al reported the salivary lactoferrin as a noninvasive biomarker for the detection of $\mathrm{AD}$ and MCI patient as compared to controls using matrix-assisted laser desorption/ionization/ time-of-flight-mass spectrometry (MALDI-TOF/TOF-MS) [26]. Analysis indicates salivary lactoferrin can be used for population screening and for identifying those underdiagnosed subjects with very early stages of mild cognitive impairment and AD. The accuracy for AD diagnosis shown by salivary lactoferrin was greater than that obtained from core CSF biomarkers. The study suggested identifying the effect of dementia on the salivary metabolite composition will improve early diagnosis. In addition, NMR based metabolic profile of saliva in MCI $(n=8)$, and AD patients ( $\mathrm{n}=9$ ), compared to healthy controls $(n=12)$ revealed the significant changes in salivary concentration of 22 metabolites including galactose, imidazole, acetone, creatine, 5-aminopentanoate and propionate, and acetone. The study demonstrated that these altered metabolites may play an important role in understanding the biochemistry of patients with MCI and AD suggesting the potential use of saliva metabolomics for the early diagnosis of AD and MCI [5]. A recent study by Huan et al in saliva using LCMS technique differentiated cognitively normal $(\mathrm{CN}), \mathrm{MCI}$, and $\mathrm{AD}$ groups. The study $(\mathrm{n}=109)$ was carried out in two phase: Discovery Phase (DP) $(\mathrm{n}=82 ; 35 \mathrm{CN}, 25 \mathrm{MCI}, 22 \mathrm{AD})$ and Validation Phase (VP) ( $\mathrm{n}=27 ; 10 \mathrm{CN}, 10 \mathrm{MCI}, 7$ AD). The analysis revealed 63 biomarkers for $\mathrm{CN}$ versus $\mathrm{AD}, 47$ for $\mathrm{AD}$ versus MCI, and 2 for MCI versus CN. They determined 3-important metabolite (methylguanosine, histidinyl-phenylalanine, and choline-cytidine) which distinguished AD from CN and MCI with the area under the curve value 1.000 while MCI and CN groups were best discriminated with 2-metabolite (glucosyl galactosyl hydroxylysine and glutamine carnitines) (DP: $\mathrm{AUC}=0.779$; VP: $\mathrm{AUC}=0.889$ ). They were able to distinguish $\mathrm{AD}$ from $\mathrm{CN}$ and MCI with good diagnostic performance using these confirmed metabolites (AUC >0.8). The study concluded that saliva as a promising bio-fluid for both unbiased and targeted AD biomarker discovery and mechanism detection [6]. Figueira et al presented a procedure for both an untargeted and targeted analysis of the saliva metabolome from patients with AD or vascular dementia using nuclear magnetic resonance (NMR) spectroscopy in combination with multivariate data analysis. The analysis identified seven statistically significant, discriminatory metabolites (histamine, succinate, taurine, acetic acid, glycerol, dimethyl sulfone, and propionate) in patients with dementia as compared to controls. Out of them, histamine, succinate, and taurine are known to be important in AD. Dimethyl sulfone and propionate originate from the diet and bacterial flora and might reflect poorer periodontal status in dementia patients. Analysis indicated the abnormalities in taurine/ hypotaurine pathway, pyruvate metabolism, glycolysis, and tricarboxylic acid (TCA) cycle. The study also suggested that the saliva metabolome has potential use for screening and early detection of dementia [27]. Pareja, et al. analyzed the saliva sample in 177 subjects including AD, PD, and age-matched controls using enzyme-linked immunosorbent assay (ELISA) kit for early diagnosis of AD. The study identified an elevated level of $\mathrm{A} \beta 42$ in the early stage of $\mathrm{AD}$ as compared to controls suggesting $\mathrm{A} \beta 42$ may be used as a potential biomarker to the early diagnosis of AD [28]. Later on, another study has also reported A $\beta$ and Tau in CSF as bio-markers in $\mathrm{AD}$ patients [29]. Liang et al. analyzed the salivary metabolome of AD patients using fast ultra-high performance liquid chromatography coupled with time-of-flight mass spectrometry (FUPLC-MS) in 256 patients and 218 age-matched controls. Sphinganine-1-phosphate, ornithine, and phenyllactic acid were identified as candidate biomarkers for early diagnosis of AD [30]. The study concluded that these metabolites can be linked to metabolic pathways which may be useful in targeting for drug discovery with the aim of finding antagonists. 


\section{Multiple sclerosis}

Multiple sclerosis (MS) is a chronic inflammatory disease of the central nervous system caused by the interaction of genetic predisposition, environmental factors, and aberrant immune response, but the pathogenesis of the disease remains unclear [31]. Clinically, the diagnosis of MS is mainly symptoms based therefore; biomarkers that can enhance symptom-based assessments and predict treatment response are greatly needed in clinical settings. In order to identify biomarkers for patients with MS, Karlík, et al. analyzed the whole saliva in 29 patients with MS and 29 healthy controls to compare the concentrations of oxidative stress markers using spectrophotometry. The study found the higher concentrations of thiobarbituric acid (oxidative stress marker) in a patient with MS as compared to controls. The study provided the potential use of saliva in monitoring the patients with MS disease [32].

\section{Conclusion}

Many neurological diseases are devastating and impose a socioeconomic burden on the individual and society. This review highlights the importance of the development of non-invasive, accessible and cost-effective diagnostic tests with the goal of early identification of neurological diseases. Evaluation of saliva is potential in developing the non-invasive diagnostic test for the discovery of disease biomarkers, but the value and applicability of saliva in biomarkers discovery for the diagnosis of neurological diseases remains in question. The existing research remains in the evolution phase in discriminating the reliability of salivary biomarkers for early detection of neurological conditions. Due to the absence of concluding results of the current studies, there is an urgent need for enhanced research efforts to conduct a non-invasive screening technique based on salivary biomarkers discovery. Appropriate saliva collection and processing protocols need to be standardized in order to reduce the biases and allow correct identification of salivary biomarkers. This review article provides a comprehensive knowledge of human saliva and its role in neurological diseases. It is essential to understand the importance of saliva as a diagnostic medium so that the study can make appropriate interpretations of how alterations in the composition of saliva are related to physiological or pathological conditions. The abundance of potential biomarker molecules in saliva make them more applicable for detection of neurological diseases. The development of advanced metabolomics techniques has shown insights toward an understanding of human saliva as a mirror reflecting our health status. In the near future, salivary biomarkers will be applied to the early detection, health care decisions, prognosis, risk assessment and monitoring of treatments with specific outcomes. For the future perspective of saliva research, the challenge is to translate large-scale information of salivary metabolomics to predict an individual's outcomes in relation to health and diseases.

\section{References}

1. Lee YH, Wong DT (2009) Saliva: an emerging biofluid for early detection of diseases. Am J Dent 22: 241-8.

2. Anker P, Mulcahy H, Chen XQ, Stroun M (1999) Detection of circulating tumor DNA in the blood (plasma/serum) of cancer patients. Cancer Metastasis Rev 18: 65-73.

3. Rieger-Christ KM, Mourtzinos A, Lee PJ, Zagha RM, Cain J, et al. (2003) Identification of fibroblast growth factor receptor 3 mutations in urine sediment DNA samples complements cytology in bladder tumor detection. Cancer 98: 737-44.

4. Wong LJC, Lueth M, Li XN, Lau CC, Vogel H (2003) Detection of mitochondrial DNA mutations in the tumor and cerebrospinal fluid of medulloblastoma patients. Cancer Res 63: 3866-71.

5. Yilmaz A, Geddes T, Han B, Bahado-Singh RO, Wilson GD, et al. (2017) Diagnostic biomarkers of Alzheimer's disease as identified in saliva using 1H NMR-based metabolomics. J Alzheimers Dis 58: 355-9.

6. Huan T, Tran T, Zheng J, Sapkota S, MacDonald SW, et al. (2018) Metabolomics Analyses of Saliva Detect Novel Biomarkers of Alzheimer's Disease. J Alzheimer's Dis 65: 1401-16.

7. Kang WY, Yang Q, Jiang XF, Chen W, Zhang LY, et al. (2014) Salivary DJ-1 could be an indicator of Parkinson's disease progression. Front Aging Neurosci 6: 1-7.

8. Zheng J, Dixon RA, Li L (2012) Development of isotope labeling LC-MS for human salivary metabolomics and application to profiling metabolome changes associated with mild cognitive impairment. Anal Chem 84: 10802-11.

9. Yakob M, Fuentes L, Wang MB, Abemayor E, Wong DT (2014) Salivary biomarkers for detection of oral squamous cell carcinoma: current state and recent advances. Curr Oral Heal Reports 1: 133-41.

10. Ilyin SE, Belkowski SM, Plata-Salamán CR (2004) Biomarker discovery and validation: technologies and integrative approaches. Trends Biotechnol 22: 411-6.

11. Silberring J, Ciborowski P (2010) Biomarker discovery and clinical proteomics. TrAC Trends Anal Chem 29: 128-40.

12. Blennow K, Dubois B, Fagan AM, Lewczuk P, de Leon MJ, et al. (2015) Clinical utility of cerebrospinal fluid biomarkers in the diagnosis of early Alzheimer's disease. Alzheimers Dement 11: 58-69.

13. Srivastava S, Love-Nichols JA, Dies KA, Ledbetter DH, Martin CL, et al. (2019) Meta-analysis and multidisciplinary consensus statement: exome sequencing is a first-tier clinical diagnostic test for individuals with neurodevelopmental disorders. Genet Med 0: 1-9.

14. Dodds MW, Johnson DA, Yeh CK (2005) Health benefits of saliva: a review. J Dent 33: 223-33.

15. Lima DP, Diniz DG, Moimaz SAS, Sumida DH, Okamoto AC (2010) Saliva: a reflection of the body. Int J Infect Dis 14: e184-8.

16. Proctor GB, Carpenter GH (2007) Regulation of salivary gland functions by autonomic nerves. Auton Neurosci 133: 3-18.

17. Proctor GB, Carpenter GH (2014) Salivary secretion: mechanism and neural regulation. Saliva Secretion Functions 24: 14-29.

18. Alexander GE (2004) Biology of Parkinson's disease: pathogenesis and pathophysiology of a multisystem neurodegenerative disorder. Dialogues Clin Neurosci 6: $259-80$.

19. Chiappin S, Antonelli G, Gatti R, Elio F (2007) Saliva specimen: a new laboratory tool for diagnostic and basic investigation. Clin Chim Acta 383: 30-40.

20. Lang AE, Lozano AM (1998) Parkinson's Disease: The first of two parts. N Engl J Med 339: 1044-53.

21. Tysnes OB, Storstein A (2017) Epidemiology of Parkinson's disease. J Neural Transm 124: 901-5. 
22. Kang W, Chen W, Yang Q, Zhang L, Zhang L, et al. (2016) Salivary total $\alpha$-synuclein, oligomeric $\alpha$-synuclein and SNCA variants in Parkinson’s disease patients. Sci Rep 6: 28143.

23. Devic I, Hwang H, Edgar JS, Izutsu K, Presland R, et al. (2011) Salivary a-synuclein and DJ-1: potential biomarkers for Parkinson's disease. Brain 134 : e178.

24. Tsuruoka M, Hara J, Hirayama A, Sugimoto M, Soga T, et al. (2013) Capillary electrophoresis-mass spectrometry-based metabolome analysis of serum and saliva from neurodegenerative dementia patients. Electrophoresis 34: 2865-72.

25. Zhuo JM, Wang H, Praticò D (2011) Is hyperhomocysteinemia an Alzheimer's disease (AD) risk factor, an AD marker, or neither? Trends Pharmacol Sci 32: $562-71$.

26. Carro E, Bartolomé F, Bermejo-Pareja F, Villarejo-Galende A, Molina JA, et al. (2017) Early diagnosis of mild cognitive impairment and Alzheimer's disease based on salivary lactoferrin. Alzheimer's Dementia: Diagn Assess Dis Monit 8: 131-8.

27. Figueira J, Jonsson P, Adolfsson AN, Adolfsson R, Nyberg L, et al. (2016) NMR analysis of the human saliva metabolome distinguishes dementia patients from matched controls. Mol Biosyst 12: 2562-71.

28. Bermejo-Pareja F, Antequera D, Vargas T, Molina JA, Carro E (2010) Saliva levels of Abeta1-42 as potential biomarker of Alzheimer's disease: a pilot study. BMC Neurol 10: 108.

29. Lee JC, Kim SJ, Hong S, Kim Y (2019) Diagnosis of Alzheimer’s disease utilizing amyloid and tau as fluid biomarkers. Exp Mol Med 51: 53.

30. Liang Q, Liu H, Zhang T, Jiang Y, Xing H, et al. (2015) Metabolomics-based screening of salivary biomarkers for early diagnosis of Alzheimer's disease. RSC Adv 5: 96074-9.

31. Lassmann H (2018) Multiple sclerosis pathology. Cold Spring Harb Perspect Med 8: a028936.

32. Karlík M, Valkovič P, Hančinová V, Krížová L, Tóthová L, et al. (2015) Markers of oxidative stress in plasma and saliva in patients with multiple sclerosis. Clin Biochem 48: 24-28. 